\title{
DE LAS TERTULIAS A LA CONSPIRACIÓN: LA DISPUTA POR LA BELLEZA Y LAS AMISTADES PELIGROSAS DE ALEJANDRO MALASPINA EN MADRID
}

\author{
Juan Manuel Sánchez Arteaga \\ Universidade Federal da Bahia \\ Email: juanma.ufba@gmail.com \\ ORCID iD: https://orcid.org/0000-0001-6266-4038
}

Recibido: 4 agosto 2020; Aceptado: 28 febrero 2021

Cómo citar este artículo/Citation: Sánchez Arteaga, Juan Manuel (2021) “De las tertulias a la conspiración: la disputa por la belleza y las amistades peligrosas de Alejandro Malaspina en Madrid", Asclepio, 73 (2): p570. https://doi.org/10.3989/asclepio.2021.28

RESUMEN: Este artículo revisa el periodo transcurrido por Malaspina en Madrid tras su "viaje alrededor del mundo", hasta su detención en noviembre de 1795. A la luz de fuentes hasta ahora ignoradas por la historiografía malaspiniana, se analiza la participación de Malaspina en una disputa literaria en el Diario de Madrid, donde llegó a publicar bajo seudónimo cuatro cartas filosóficas sobre la belleza que constituyen un antecedente directo de su Meditación filosófica, su obra más personal, escrita en presidio tras su arresto. Por último, se analiza la relación de Malaspina con su vecina madrileña Fernanda O'Connock, marquesa de Matallana, mujer vinculada a la oposición política a Godoy y destacada agente conspiradora contra el primer ministro en dicho periodo, quien muy posiblemente tuvo un papel mucho más activo de lo que hasta ahora se le había reconocido en la conspiración Malaspina.

PALABRAS CLAVE: Alejandro Malaspina; Diario de Madrid; Ciriaco de Ceballos; Marquesa de Matallana; Oposición a Godoy; Estética.

\section{FROM GATHERINGS TO CONSPIRACY: THE DISPUTE ABOUT BEAUTY AND ALEJANDRO MALASPINA'S DANGEROUS LIAISONS IN MADRID}

ABSTRACT: This article reviews the period spent by Malaspina in Madrid after his "voyage around the world", until his arrest in November 1795. Exploring sources hitherto ignored by Malaspinian historiography, the article focuses on Malaspina's participation in a literary dispute which took place in the Diario de Madrid, where he published under pseudonym four philosophical letters on Beauty. These letters constitute the direct antecedent of his Meditation on Beauty, his most personal work, written in prison after his arrest. Lastly, the paper analyses Malaspina's relationship with his neighbour in Madrid, Fernanda O'Connock, Marchioness of Matallana, a woman linked to the political opposition to Manuel Godoy and an outstanding conspirator agent against the Prime Minister, who very possibly played a much more active role than previously recognised in the so-called "Malaspina conspiracy".

KEY WORDS: Alejandro Malaspina; Diario de Madrid; Ciriaco de Ceballos; Marchioness of Matallana; Opposition to Godoy; Aesthetics.

Copyright: ( 2021 CSIC. Este es un artículo de acceso abierto distribuido bajo los términos de la licencia de uso y distribución Creative Commons Reconocimiento 4.0 Internacional (CC BY 4.0) 


\section{EL RETORNO DEL HÉROE: MALASPINA EN MADRID}

Si bien para la marinería y para muchos de los oficiales que acompañaron a Malaspina en su gran expedición científica, su periplo alrededor del mundo había finalizado con éxito al arribar al puerto de Cádiz en septiembre de 1794, después de 62 meses de trabajo incansable a bordo de las corbetas Atrevida y Descubierta, la perspectiva abierta tras la llegada a España era bien diferente para el italiano y para un grupo escogido de sus hombres. En realidad, para Malaspina, comenzaba ahora el momento crucial de su carrera. La expedición comandada por él no había dejado, ni un solo día, de producir todo tipo de materiales científicos e informes político-económicos de la más alta calidad. Ahora había que ordenarlos y seleccionar lo que debía ser publicado de entre un conjunto inmenso de datos que comprendía series de mediciones geodésicas, mapas y cartas hidrográficas, láminas, cuadros y dibujos realizados en las diversas etapas del periplo, así como decenas y decenas de cajas con las que probablemente eran las más completas colecciones ${ }^{1}$ botánicas, zoológicas, mineralógicas y etnográficas del momento ${ }^{2}$. Se trataba ahora de poner en orden toda esa cantidad abrumadora de información y de material para emprender el proyecto gigantesco de la edición de los diarios y de los Atlas derivados del viaje.

El italiano, acompañado por alguno de sus oficiales, naturalistas y pintores ${ }^{3}$, se desplazó enseguida hasta Madrid para poner manos a la obra. Paralelamente -según se desprende de su correspondenciaen esa época Malaspina comenzó a dar muestras de sentirse obsesionado por las circunstancias políticas y por la puesta en marcha de un conjunto de reformas radicales en la administración del gobierno y los territorios coloniales, reformas que el italiano consideraba necesarias para salvar a la monarquía ${ }^{4}$. A partir de su privilegiada experiencia estudiando las colonias en todos sus aspectos -naturales, económicos, sociales...-, había vuelto a la metrópoli convencido que "el código antiguo de legislación de América no podía subsistir"5. De esta forma, nada más desembarcar en la península y a la vez que intentaba organizar las tareas para publicar el viaje, Malaspina pensaba en las formas de maniobrar políticamente en Madrid para remodelar el gobierno y, con ello, regenerar la monarquía y salvar el Imperio colonial español. En algunas de las cartas privadas remitidas por Malaspina desde Cádiz, antes aún de trasladarse a Madrid, el italiano confesó a su amigo Greppi que tenía en la cabeza un proyecto de reforma política de la monarquía, y que trataría de llevarlo a cabo a pesar de los altísimos riesgos que eso implicaba: en este momento la vida es un puro juego del que no debemos preocuparnos mucho (...). Si no me engañan las experiencias de cuatro largos años, puedo decir que he atado aquellos pocos cabos sueltos con los que se deba restablecer la prosperidad o, mejor dicho, la regeneración de la monarquía ${ }^{6}$.

Una vez en Madrid, el 7 de diciembre de $1794 \mathrm{Ma}-$ laspina fue recibido con todos los honores por la familia real'. Malaspina pensaba ya en encontrar un modo seguro de convencer al rey de que lo más sensato, por el bien de la propia corona, era emprender una profunda reforma política y administrativa en las colonias. El plan de Malaspina implicaba la renuncia a extender los límites territoriales del Imperio y a imponer la soberanía española por la fuerza sobre pueblos indígenas aún no sometidos a la dominación monárquica:

Fijados ya los límites del imperio (...) es justo examinar, en la inmensidad de países que aún quedan, cuáles son los que forman una parte efectiva de la Monarquía, gobernados ya por nuestras leyes y capaces de contribuir en algún modo a la defensa de la república, y cuáles los que no debemos considerar sujetos a la autoridad nuestra ${ }^{8}$.

A su vez, Malaspina se sentía obligado a plantear al rey la necesidad de abandonar los principios "torcidos, injustos y perniciosos" que habían definido la administración imperial hasta entonces, y establecer, mediante una nueva constitución regida por el principio confederal, "un nuevo plan de derecho público" para las colonias americanas y asiáticas, que, unificado bajo la corona borbónica, aglutinara a una comunidad de naciones autónomas, independientes y "emancipadas" ${ }^{\prime \prime}$. Malaspina estaba convencido de que esa nueva confederación hispánica abriría un espacio político, económico, y social de convivencia, de intercambio comercial y cultural pacífico y justo, y de enriquecimiento mutuo entre la metrópoli y sus antiguos territorios coloniales. La transformación del decadente imperio español en una confederación de naciones libremente asociadas bastaría para, de un lado, liberar a España "de los pesados grillos que le causan las posesiones de ultramar y [de otro lado], presentarle un plan general de reunión [con las colonias], con el cual sean todas felices y no teman las invasiones externas, ni apetezcan las riquezas ajenas" ${ }^{10}$.

Todas las claves para esa soñada regeneración, que Alejandro pensaba haber juntado, pasaban, a su juicio, por la destitución del primer ministro Manuel Godoy. Ante la caótica situación política en Madrid, Malaspina se mostraba cada vez más preocupado y convencido de que el mal gobierno del Imperio -personificado en 
el valido Godoy, a quien Malaspina calificaba de sultán en sus cartas privadas- había llegado a un grado tal de abyección que ya no era posible evitar el derramamiento de "la sangre de los pobres". Poco después de ser presentado a los reyes, le confesaba por carta a su amigo Greppi:

Un único día me habría bastado para mostrar mi sistema. Lo he visto todo y lo he visitado todo. Puede ser que en el caos del sistema actual haya sólo un pequeño paso del buen al mal camino, de la sinrazón a la sana filosofía. Todo parecía favorable (...) pero el acceso al sultán es tan difícil, y todo lo que le rodea está [tan] hundido en la confusión y en la inacción, que es imposible hacerse entender y poder actuar ${ }^{11}$.

Durante su estancia en la capital de España, Malaspina participó asiduamente en diversas tertulias, en las que se mostraba cada vez más incapaz de disimular su frustración ante la ineptitud política del primer ministro ${ }^{12}$. En medio de estas frustraciones, abrumado por la nostalgia de la acción y por el oprobio de la corrupción que se respiraba en la corte, Malaspina reconocía sentirse paralizado en Madrid, "condenado al triste oficio de autor"13. La altura moral de sus ideales parecía convivir en estos momentos desesperantes -en los que, por si fuera poco, veía paralizados todos sus planes originales para la publicación de su viaje ${ }^{14}$. con una "insanable comezón política" ${ }^{15}$. Durante este periodo, el italiano, convencido de tener la clave para "salvar a la patria", no consiguió substraerse a una especie de obsesión mesiánica que, a la postre, acabaría haciéndolo naufragar en manos del primer ministro ${ }^{16}$, pagando un altísimo precio.

\section{LA DISPUTA POR LA BELLEZA}

En los pocos momentos de asueto que restaban a Malaspina durante su estancia en la corte madrileña y mientras trataba de organizar la publicación del viaje, el italiano tomó afición por las tertulias eruditas que estaban de moda entre las clases altas de la capital de España. Durante su estancia en Madrid, por las mañanas, Malaspina solía juntarse para conversar sobre el devenir de sus trabajos y otros asuntos con sus hombres y otros personajes notables del reino. Esas reuniones matutinas acontecían en casa del cónsul sueco Juan Jacobo Gahn, quien vivía en Cádiz, pero poseía una residencia en la capital. Por las tardes, estas mismas personas se reunían para departir amigablemente sobre asuntos mundanos en el paseo del Prado ${ }^{17}$. Y por las noches, Malaspina acudía a la tertulia erudita que se celebraba en la casa del ministro de Marina Antonio Valdés ${ }^{18}$, su antiguo protector ${ }^{19}$. En una de aquellas reuniones en casa del bailío Valdés -tertulia a la que también asistieron el clérigo Manuel $\mathrm{Gi}^{20}$, los pintores de la expedición, Juan Ravenet y Fernando Bambrila ${ }^{21}$ y el joven discípulo de Alejandro, Fabio Ala Ponzone ${ }^{22}$ se estableció una batalla dialéctica entre Malaspina y el santanderino Ciriaco de Ceballos, uno de los más brillantes oficiales científicos que habían participado en la expedición transoceánica dirigida por el italiano. La disputa inicial parece haberse centrado en torno a la cuestión de si las mujeres de ciertas naciones podían considerarse más bellas que las de otras por motivos naturales, no dependientes del capricho, la costumbre o las modas. Malaspina defendía firmemente que sí. La superior belleza de los pueblos europeos era causada por la propia naturaleza y, por tanto, no dependía de modas ni de costumbres. Por su parte, Ceballos mostró una firme oposición al eurocentrismo de Malaspina, negando que hubiese unos pueblos esencialmente bonitos, y otros inherentemente feos, y afirmando que la idea de lo bello dependía de su historia y sus costumbres y que, en consecuencia, no podía afirmarse que las mujeres europeas fuesen más hermosas que las de otras latitudes. Cabe recordar que, a lo largo de la Expedición Malaspina, Ceballos había quedado fascinado por la hermosura de algunos de los pueblos contactados (a los que no dudaría posteriormente en atribuir una belleza igual o superior a la de los europeos en sus cartas al Diario de Madrid). Sabemos que, por ejemplo, durante su estancia en Nutka, en Canadá, una "helada región" cercana al polo, Ceballos se impresionó profundamente por la belleza de una de las nativas, la joven esposa del cacique Macuina ${ }^{23}$. Esa admiración hacia la belleza de otros pueblos también se extendió en algún caso a su organización social. Durante su estancia en Tonga, Ceballos quedó tan impresionado por su experiencia en la isla, que prometió al cacique local, Vuna, que tras su retorno a España regresaría para pasar allí el resto de sus días. En reconocimiento, Vuna nombró a Ceballos su "hijo adoptivo", con rango de príncipe ${ }^{24}$. Por lo demás, puede inferirse que una parte significativa de los contactos "antropológicos" de Ceballos con informantes nativos durante el viaje alrededor del mundo se produjeron -al menos en ciertas etapas de la expedición- sobre todo, con "informantes nativas", tal y como parece indicar el vocabulario que preparó en la isla de Tonga, repleto de alusiones a la anatomía femenina ${ }^{25}$. Tal vez con la memoria de aquellas experiencias en la cabeza, Ceballos rechazó uno por uno los argumentos que Malaspina expuso en aquella tertulia cortesana, y defendió con ahínco que no existían criterios absolutos de belleza, sino que estos criterios dependían completamente de las costumbres de cada nación. 
A partir de ese "duelo dialéctico" entre ambos tertulianos, la discusión fue reconducida en términos más filosóficos a la cuestión general de si lo bello era una propiedad esencial de la naturaleza o si, por el contrario, era tan solo un resultado caprichoso y variable de las modas y costumbres. Espoleado por aquella discusión con Ceballos, y en un momento en que los trabajos de publicación de su viaje científico se encontraban paralizados, Malaspina decidió escribir un texto didáctico sobre lo bello en la prensa madrileña y, con ánimo verdaderamente ilustrado, algunos días después de aquella tertulia publicó una primera carta anónima sobre la belleza en el Diario de Madrid, donde, rememorando su polémica estética con Ceballos -a quien caracterizó cómicamente como un tertuliano de desmesurado corbatín- el italiano defendió su postura con argumentos filosóficos ${ }^{26}$. A pesar de no haber firmado aquella carta, la autoría del italiano fue reconocida inmediatamente por el resto de contertulios. Así que no pasó mucho tiempo antes de que el texto del italiano fuera replicado por escrito en el mismo diario por Ciriaco de Ceballos, quien intentó refutar, uno por uno, los argumentos de su superior. El italiano, a su vez, recogió el guante y dio su contrarréplica a Ceballos, quien por su parte volvió a responder originando nuevas contestaciones de Alejandro a sus argumentos relativistas. A la postre, otros miembros de la expedición que habían participado en la tertulia del ministro Valdés se unieron a la polémica publicando sus propias cartas. Entre ellos estaban Juan Ravenet, Fernando Bambrila y Fabio Ala Ponzone (el único de entre los expedicionarios que salió en defensa de Malaspina). También Manuel Gil -que por esas fechas había entablado una indisimulable enemistad con Malaspina-, se sumó a la disputa, publicando varias cartas en las que criticó con sorna tanto las ideas estéticas del italiano cuanto su deficiente empleo del castellano, dando origen a las contribuciones más sarcásticas en el debate. Con posterioridad, además de los contertulios que estuvieron presentes en casa del ministro Valdés, la marquesa de Matallana -quien por entonces se había convertido en íntima amiga de Malaspina- remitió al Diario de Madrid otra misiva para intervenir en la polémica.

Aunque las cartas de Malaspina al Diario de Madrid fueron firmadas con diferentes seudónimos, en ellas usó exactamente los mismos argumentos y ejemplos que, tiempo después, ya en la cárcel, retomó en su Meditación sobre lo bello, lo que ha posibilitado la identificación de estos textos malaspinianos. En total,
Malaspina publicó cuatro cartas en el Diario de $\mathrm{Ma}$ drid. Si mi análisis es correcto, debe descartarse el texto que Belén Fernández y Dario Manfredi atribuyeron incorrectamente a Malaspina dentro de esta disputa ${ }^{27}$, aunque estos autores sí identificaron correctamente una de las cartas con las que el clérigo Manuel Gil participó en la polémica ${ }^{28}$. Sin embargo, no fue realizado un cotejo exhaustivo del contenido de todas cartas sobre la belleza publicadas en el Diario de Madrid con el texto de la Meditación filosófica sobre de lo bello en la naturaleza, que Malaspina comenzó a escribir estando ya en prisión, y cuya primera edición impresa solo apareció en $2007^{29}$, diez años después del artículo de Fernández y Manfredi. Comparando la Meditación filosófica con las cartas del Diario de Madrid, pueden atribuirse con total confianza a Malaspina cuatro cartas publicadas a lo largo de 1795 (véase TABLA 1). Sus enormes coincidencias ideológicas y temáticas con la Meditación filosófica evidencian que todos esos escritos pertenecen al mismo autor. Además -por numerosos indicios que no cabe detallar aquí- pueden identificarse otros autores en el seno de la Disputa. Todos los contendientes utilizaron diferentes seudónimos, aunque alguno de los empleados apenas conseguía esconder la verdadera identidad de sus autores. El caso del santanderino Ciriaco de Ceballos es especialmente evidente, por componer sus seudónimos cambiando el orden de las letras de su nombre y apellidos, firmando como Isidro Calle Boceca y como Cesáreo Cid Cabillo, anagramas perfectos de su verdadero nombre. Por su parte, los pintores Juan Ravenet y Fernando Bambrila firmaron con las iniciales RxF, fingiendo ser los "asesores de una pintora"; el discípulo de Malaspina Fabio Ala Ponzone firmó sus cartas como Favonio y el Discípulo y el Padre Manuel Gil firmó como Gil Gilete. En el caso de la Marquesa de Matallana (la Defensora de la belleza), las claras alusiones ad hominem que otros contendientes realizaron en sus réplicas al "cortejo" de Malaspina a la marquesa posibilitan la atribución con verosimilitud. En conjunto, la disputa literaria sobre la belleza en el Diario de Madrid comprende 33 cartas y se extendió durante prácticamente todo 1795 (véase la TABLA 1). Finalizó abruptamente en noviembre de aquel mismo año, cuando Malaspina fue detenido y encarcelado, acusado de organizar un complot contra Godoy ${ }^{30}$. En lo que sigue de este trabajo pretendo dar una reinterpretación a estos hechos, en los que, hasta el momento, la historiografía malaspinista no había otorgado el suficiente protagonismo a una de las personas más próximas a Malaspina en aquellos tiempos, y que sin embargo tuvo un papel fundamental (y mucho más activo de lo que hasta ahora se creía) en la "conspiración Malaspina". 


\begin{tabular}{|c|c|c|c|c|}
\hline & $\begin{array}{l}\text { Fecha de publicación en } \\
\text { el Diario de Madrid: }\end{array}$ & $\begin{array}{c}\text { (Número): Páginas } \\
\text { del Diario de } \\
\text { Madrid }\end{array}$ & Seudónimo: & Autoría atribuida ${ }^{2}:$ \\
\hline -CARTA N. 1 & $17 / 10 / 1794$ & (290):1183-85 & Sin firmar & Pedro Estala \\
\hline -CARTA N. 2 & $30 / 10 / 1794$ & (303):1239-41 & El Mismo. & Pedro Estala \\
\hline -CARTA N.o 3 & $27 / 04 / 1795$ & (113):481-2 & Sin firmar & Alejandro Malaspina \\
\hline -CARTA N. 04 & $\begin{array}{l}4.1(30 / 04 / 1795) \\
4.2(02 / 05 / 1795) \\
\end{array}$ & $\begin{array}{l}\text { (120): } 493-5 \\
(122): 501-3\end{array}$ & Isidro Calle Boceca & Ciriaco de Ceballos. \\
\hline -CARTA N. 5 & $7 / 05 / 1795$ & $(127): 521-23$ & El Censor Mensual & Pedro Estala \\
\hline -CARTA N.9 6 & $10 / 06 / 1795$ & (161): 657-58 & El Anónimo & Alejandro Malaspina \\
\hline -CARTA N. 7 & $\begin{array}{l}7.1(23 / 06 / 1795) \\
7.2(24 / 06 / 1795)\end{array}$ & $\begin{array}{l}\text { (173): 705-07 } \\
\text { (174): 709-11. }\end{array}$ & Cesáreo Cid Cabillo & Ciriaco de Ceballos \\
\hline -CARTA N.ㅇ 8 & 07/07/1795 & (188): 765-68 & El Censor Mensual & Pedro Estala \\
\hline -CARTA N. 99 & $12 / 07 / 1795$ & (193): 785-87 & Bruneti Sarnism & Ciriaco de Ceballos \\
\hline -CARTA N. $\mathrm{o} 10$ & $18 / 07 / 1795$ & (202): 809-11 & El Censor Mensual & Pedro Estala \\
\hline -CARTA N. 11 & $\begin{array}{l}11.1(23 / 07 / 1795) \\
11.2(24 / 07 / 1975)\end{array}$ & $\begin{array}{c}(307): 829-31 \\
(308): 833-835\end{array}$ & Trinepos Boceca & Ciriaco de Ceballos \\
\hline -CARTA N.o 12 & $\begin{array}{l}12.1(28 / 07 / 1795) \\
12.2(29 / 07 / 1975) \\
12.3(30 / 07 / 1795)\end{array}$ & $\begin{array}{l}(209): 849-51 \\
(210): 853-55 \\
(211): 857-59\end{array}$ & $\begin{array}{l}\text { La Defensora de la } \\
\text { Belleza. L.F.A.F. }\end{array}$ & $\begin{array}{c}\text { Alejandro Malaspina (vero- } \\
\text { símilmente, en coautoría con } \\
\text { Fernanda O'Connock, Marque- } \\
\text { sa de Matallana) } \\
\end{array}$ \\
\hline -CARTA N. 13 & 05/08/1795 & $(217): 880-83$ & Bocequilla & Ciriaco de Ceballos \\
\hline -CARTA N. 14 & $\begin{array}{l}14.1(06 / 08 / 1795) \\
14.2(07 / 08 / 1795) \\
\end{array}$ & $\begin{array}{l}(218): 885-86 \\
(219): 889-90\end{array}$ & El Censor Mensual & Pedro Estala \\
\hline -CARTA N.o 15 & $\begin{array}{l}15.1(10 / 08 / 1795) \\
15.2(11 / 08 / 1795)\end{array}$ & $\begin{array}{l}(222): 901-03 \\
(223): 909-11\end{array}$ & $\begin{array}{l}\text { Madrileña fea/El } \\
\text { Susodicho }\end{array}$ & Manuel Gil \\
\hline -CARTA N.o 16 & $\begin{array}{l}16.1(14 / 08 / 1795) \\
16.2(15 / 08 / 1795)\end{array}$ & $\begin{array}{l}\text { (226): } 921-23 \\
(227): 925-27\end{array}$ & $R x F$ & $\begin{array}{l}\text { Juan Ravenet y Fernando Bam- } \\
\text { brila }\end{array}$ \\
\hline -CARTA N.o 17 & $(21 / 08 / 1795)$ & $(233): 949-51$ & La Chinilla & Ciriaco de Ceballos \\
\hline -CARTA N.o 18 & 23/08/1795 & (235):957-59 & El Anciano & $\begin{array}{c}\text { Desconocida } \\
\text { (¿Antonio Valdés?) }\end{array}$ \\
\hline -CARTA N. 19 & $\begin{array}{l}19.1(29 / 08 / 1795) \\
19.2(30 / 08 / 1795)\end{array}$ & $\begin{array}{l}(241): 981-84 \\
(242): 985-87 \\
\end{array}$ & Don Simplicio & Manuel Gil \\
\hline -CARTA N.o 20 & 03/09/1795 & (246):1001-03 & La Chinilla & Ciriaco de Ceballos \\
\hline -CARTA N.o 21 & 04/09/1795 & $(247): 1005-06$ & El Expresado & Ciriaco de Ceballos \\
\hline -CARTA N.o 22 & 09/09/1795 & (252):1025-26 & El Censor Mensual & Pedro Estala \\
\hline -CARTA N. 23 & $23.1(10 / 09 / 1795)$ & $\begin{array}{l}(253): 1025-26 \\
(254): 1029-31\end{array}$ & $\begin{array}{l}\text { El hombre del chale- } \\
\text { quillo y corbatón }\end{array}$ & $\begin{array}{c}\text { Ciriaco de Ceballos }{ }^{3} \\
* \\
\text { ¿Alejandro Malaspina? } \\
\text { (apud Fernández y Manfredi, } \\
\text { 1998) } \\
\end{array}$ \\
\hline
\end{tabular}

1 Las cartas de Alejandro Malaspina están marcadas con negrilla. Todas estas cartas están accesibles en el portal electrónico de la Hemeroteca digital (Biblioteca digital Hispánica), bajo la entrada Diario de Madrid, 1788. http://hemerotecadigital.bne.es/results. vm?q=parent\%3A0001510462\&s=4100\&lang=es (acceso: 04/08/2020).

2 Los criterios específicos para la atribución de la autoría de cada una de las cartas de la disputa en el Diario de Madrid -imposibles de incluir en el presente artículo por motivos de espacio- están explicados con detalle en un libro que aborda los escritos estéticos de Malaspina y contiene una transcripción de todas las cartas de la disputa, junto a una nueva edición de su Meditación filosófica. El libro, titulado Lo bello en la naturaleza, será publicado próximamente en la colección "Estudios sobre la ciencia" del CSIC.

3 La autoría de esta carta corresponde a Ciriaco de Ceballos, a pesar de que el texto había sido atribuido previamente a Malaspina por Fernández y Manfredi (1998). Para más detalles, remito al libro Lo bello en la naturaleza (véase nota 2). 


\begin{tabular}{|c|c|c|c|c|}
\hline \multicolumn{1}{|c|}{ TABLA 1: SUMARIO DE LAS CARTAS DE LA DISPUTA SOBRE LA BELLEZA PUBLICADAS EN EL DIARIO DE MADRID (Oct. 1794- } \\
Nov. 1795)
\end{tabular}

\section{AMISTADES PELIGROSAS: LA MATALLANA Y LA "OPOSICIÓN INTERNA" A GODOY}

Por supuesto, Malaspina tenía muchísimo trabajo más allá de defender su postura eurocéntrica frente a Ceballos en aquel divertimento literario del Diario de Madrid, que a la postre acabó transformándose en un verdadero "baile de máscaras" donde los diferentes contertulios jugaban a disfrazar sus ataques protegidos por seudónimos. En realidad, la disputa puede ser entendida como una especie de descanso para la frenética actividad que caracterizaba por entonces el día a día del italiano. Y ese trabajo que consumía ahora todas las energías de Malaspina tenía una doble faz: científica y política. Durante este periodo clave, y mientras que Malaspina, coordinando su equipo de oficiales y pintores en Madrid, se afanaba, a pesar de las inmensas dificultades, en organizar los resultados de la expedición para darlos a la imprenta, el italiano se había convertido en el centro de atención de la corte española, que había recibido con verdadera expectación la vuelta del ilustrado viajero tras su expedición científica alrededor del mundo. "La variedad y amenidad de su erudición, sus costumbres y otras circunstancias, hacían su trato muy apreciable" ${ }^{31}$ en los salones ilustrados que frecuentaba en Madrid. Malaspina se veía con frecuencia y tenía bastante intimidad de forma pública con muchas personas de entre las de mayor rango de la corte y, en ese proceso, rápidamente entró en contacto con las máximas instancias de gobierno y con personajes muy próximos a los reyes. Pero al mismo tiempo, Malaspina estableció peligro- sos vínculos de amistad con miembros de la "oposición interna" 32 al gobierno de Godoy. Entre estos últimos, creó un estrecho (y fatídico) vínculo de amistad con una de sus vecinas en Madrid, Fernanda O'Connock, marquesa de Matallana, quien desde hacía meses se afanaba de forma intensa y secreta en la intriga cortesana para derrocar al valido.

La marquesa de Matallana estaba estrechamente vinculada a la llamada "oposición interna" a Godoy. Caracterizada por una ideología monárquica reformista, esta oposición política al gobierno estaba formada por personas próximas a los reyes -miembros de la nobleza, altos mandos militares y otros aristócratas progresistas y liberales, como la propia marquesa-, que se agrupaban en torno al llamado partido aragonés y, en muchos casos, a la incipiente masonería españo$\mathrm{la}^{33}$. El partido Aragonés era el partido del Conde de Aranda -primer ministro de Carlos III y fundador de la logia masónica del Gran Oriente Español-, antagonista del Conde de Floridablanca, en torno a cuya figura se agrupaba otro "partido", los Ilamados golillas. En este duelo de aragoneses y golillas, el partido aragonés se constituyó gradualmente como una fuerza de oposición al poder establecido. Aranda había intentado un programa de reformas internas cuando era primer ministro, con las que pretendía descentralizar el poder en beneficio de los Consejos. El pánico que se propagó en la corte española tras el asalto de Las Tullerías en París, en agosto del 92 y el ascendiente que sobre los reyes había adquirido Godoy (paradójicamente, un miembro del Partido Aragonés), provocaron la sustitución 
de Aranda por el Conde de Floridablanca. Después de esto, Aranda y los aragoneses no cesaron en su empeño para derribar a Godoy, de forma cada vez más notoria. Proponían una reorganización institucional de la monarquía, como contrapunto reformista a la revolución que había estallado en Francia. En 1.794 Aranda fue desterrado a Jaén tras un encontronazo con el valido, lo que provocó que sus seguidores continuasen aun con más ahínco haciendo propaganda anti Godoy. En este momento ya se conocía al grupo de los aragoneses como el partido de la oposición ${ }^{34}$. En definitiva, los aragoneses pretendían a toda costa imponer una regeneración política que, tal y como pensaba Malaspina, debía necesariamente comenzar por la inminente sustitución del valido Godoy al frente del gobierno.

Entre los miembros de la aristocracia afines al partido aragonés más activos en la tentativa de derrocar al primer ministro y más próximos a los monarcas estaba, precisamente, Fernanda O'Connock, con quien Malaspina estableció una estrechísima amistad tras instalarse en Madrid en una casa aledaña a la de la marquesa ${ }^{35}$. Era la marquesa un personaje notorio entre la aristocracia madrileña por no esconder sus ideas contra el valido y por defender abiertamente su opinión en tertulias ideológico-políticas frecuentadas por los más importantes líderes de la oposición interna, incluido el mismo Conde de Aranda ${ }^{36}$. O'Connock, "además de ser amiga estrechísima de Malaspina, mantenía públicamente discursos desmedidos sobre el gobierno y contra el ministro [Godoy] ${ }^{37 \prime \prime}$. Todo ello le había hecho ganar fama de mujer "inmoderadamente proclive a las intrigas" ${ }^{38}$. No era una reputación enteramente inmerecida, pues de hecho -a pesar de que hasta ahora había pasado inadvertido para la historiografía malaspinana- la marquesa había sido un personaje central en la organización clandestina del llamado motín de San Blas, una intentona fallida de derribar al todopoderoso primer ministro a inicios de 1795 , ya con Alejandro instalado en la corte como su vecino.

Aunque Godoy consiguió abortar in extremis el motín de San Blas, también conocido como "conspiración de Picornell", el complot -vinculado directamente al Partido Aragonés y a la masonería- se había organizado en sigilo a partir de una de las tertulias frecuentadas por la Matallana -como era conocida la marquesa-, en la que esta actuó en secreto como intermediaria entre la cabeza visible del golpe, Juan Picornell -un subversivo abogado y pedagogo mallorquín- y poderosos aristócratas de la oposición interna, que lo apoyaban entre bastidores. Desde su llegada a la corte, el abogado Juan Picornell procuró buscar nuevos partidarios para su causa, y la primera que se declaró tal fue la marquesa de Matallana, en una tertulia que comenzó a concurrir con ocasión de defenderla un pleito (...). En la expresada tertulia no se hablaba de otra cosa sino de la revolución proyectada, [y] en ella fue donde se hicieron partidarios el conde de Aranda, el duque de Almodóvar, el conde de Tepa y otras muchas personas principales del Reino, de modo que la expresada marquesa de Matallana hizo más recluta que todos los demás partidarios ${ }^{39}$.

Finalmente, Picornell -que en un principio fue detenido y condenado a la horca-, consiguió fugarse de España con ayuda de sus poderosos y secretos amigos. Por su parte, la astuta Matallana salió indemne del caso sin levantar sospechas, y continuó ejercitando su "incontenible propensión a la intriga" en las tertulias políticas de la corte donde, dada su proximidad física e ideológica, acabó inevitablemente coincidiendo con su vecino Malaspina, frecuentador de tertulias, quien, como la marquesa, tampoco se esforzaba mucho en disimular su repulsión ante la política de Manuel Godoy.

\section{DEL “CORTEJO” LITERARIO A LA PRISIÓN Y EL EXILIO}

Nada más conocer a Malaspina, la marquesa de Matallana debió de quedar favorablemente impresionada por el Brigadier quien -además de un hombre exitoso, cultísimo, atractivo, soltero y recién entrado en la cuarentena-, había sido recibido a su retorno de la expedición científica como un héroe nacional. Rápidamente se estrechó una amistad íntima entre ambos, hasta el punto de llegar a suscitar chascarrillos en los círculos cortesanos y entre las personas cercanas a Alejandro, que inclusive se vieron reflejados en la Disputa sobre la belleza en el Diario de Madrid ${ }^{40}$. Por estas fechas, todo el mundo en la corte sabía (y comentaba) que el italiano era el nuevo cortejo de la marquesa ${ }^{41}$. De acuerdo con las costumbres imperantes entre la aristocracia madrileña de fin de siglo, esa situación les daba licencia para pasar muchas horas juntos en total privacidad, a pesar de la Matallana ser una mujer casada, pues los cortejos resultaban perfectamente admitidos entre las clases altas de Madrid. Así describía esta costumbre el británico Joseph Towsend, de visita en Madrid entre 1786 y 1787 :

Cuando uno va a visitar a una dama -pues, habiendo una dama en la casa la visita es para ella-, no ha de llamar a la puerta ni preguntarle nada al portero, sino que debe encaminarse directamente a la habitación en la que ella suele recibir a sus visitantes (...). Los amigos son en su mayoría caballeros, pues las damas rara vez hacen una visita que no sea de ceremonia, y entre esos 
amigos reunidos allí uno de ellos es por lo común "el cortejo" (...) a lo largo de mi estancia en España no oí jamás mencionar a un marido celoso ni llegué a saber con certeza que tal cosa existiera (...) El marido tiene tan poca importancia en el hogar y se le ve tan poco por allí, y cuando se le ve es un extraño tan completo para todas las visitas de su familia, que al amante le resulta muy fácil pasar inadvertido (...). Este debe estar presente a cualquier hora del día ya sea en público o en privado, ya esté enferma la dama o goce de buena salud y debe ser invitado a todas partes para acompañarla ${ }^{42}$.

Como es sabido, en noviembre de 1795 , la marquesa de Matallana actuó como amanuense de Malaspina transcribiendo sus planes para derrocar al gobierno de Godoy sin que la letra y el peculiar castellano del italiano le delatasen. Pues bien, en Julio de aquel mismo año, es decir, pocos meses antes, la marquesa ya había realizado una labor semejante como amanuense de su amigo Brigadier en la Disputa sobre la belleza, enviando al Diario de Madrid una larga carta bajo seudónimo, que con toda probabilidad fue dictada por Malaspina, con adicciones de la propia marquesa ${ }^{43}$. El texto posee un conjunto de coincidencias muy específicas con la Meditación filosófica, las cuales, a mi juicio, hacen indudable su atribución al italiano. La carta contiene toda una serie de ideas y expresiones muy parecidas o idénticas a las que aparecen en la Meditación, escrita por Malaspina durante su confinamiento. Entre los rasgos coincidentes con la Meditación filosófica que refuerzan la autoría malaspiniana de la carta están: el uso de vocabulario marinero y referencias a la arquitectura naval; citas del filósofo Condillac; una defensa del papel de las mujeres en la sociedad; la defensa de una visión histórica cíclica y degeneracionista, claramente influida por Vico y Filangieri; el empleo de referencias a la música de Haydn como paradigma de un arte desnaturalizado, que se ha olvidado de imitar a la naturaleza, etc. Por si fuera poco, en la carta de la Defensora se hace una mención explícita al viaje de Malaspina al archipiélago de Vavao (autocita que el italiano repite en su Meditación), y se incluyen referencias antropológicas sobre algunos de los pueblos americanos contactados durante la expedición y otros grupos étnicos que sólo un erudito viajero con alto interés en asuntos etnográficos, como el propio Malaspina, podía tener en el Madrid de la época. La hipótesis de la coautoría con la Matallana se basa en las múltiples alusiones al cortejo en las réplicas a Malaspina por parte del resto de los contendientes y en que, a diferencia de la propia Meditación y del resto de cartas de Malaspina en el Diario de Madrid, el texto de la defensora de la belleza carece de los defectos formales característicos del castellano escrito de Malaspina. Podemos pensar que el italiano buscase en la marquesa la ayuda de alguien que pudiese corregir los defectos de su escritura sin tener que sufrir la insoportable censura que ejercía sobre sus escritos el padre Gil (y sin dejar rastros de su letra). Resulta verosímil pensar que ambos escribieron conjuntamente la carta de la Defensora, de forma semejante a como hicieron al redactar el plan para derrocar a Godoy en noviembre de aquel año, cuando la Matallana volvió a actuar como amanuense para Alejandro. El 28 de julio de 1795 fue publicada la primera entrega del escrito -la carta continuó a lo largo de los dos días siguientes, 29 y 30 de julio-, que venía firmado por una misteriosa dama madrileña, la Defensora de la belleza. La carta pretendía sumar argumentos a los que habían sido expuestos anteriormente por Malaspina en su primera carta anónima enviada al Diario de Madrid, donde el italiano había defendido la existencia de una belleza esencial en la naturaleza. La carta de la Defensora era una contrarréplica dirigida a Ciriaco de Ceballos, quien había refutado en el diario las eurocéntricas tesis estéticas de Malaspina, utilizando como seudónimo el anagrama de su nombre, Isidro Calle Boceca.

Fig. 1. Portada del Diario de Madrid que contenía la primera entrega de la carta firmada por La Defensora de la belleza (coescrita por la marquesa de Matallana y Malaspina) en réplica a las cartas anteriores de Isidro Calle Boceca (Ciriaco de Ceballos). Reproducido con permiso de la Biblioteca Nacional de España.

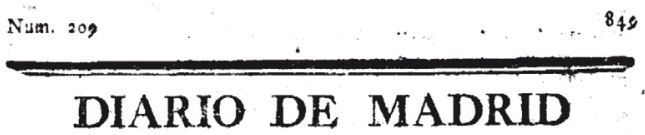

DRL MARTES 23 DE JULIO DE 1795 .

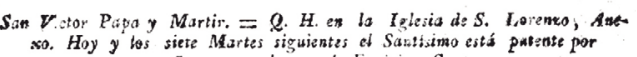
so. Hoy y tos siete Martes siguientes el Santisimo está pateote por
mañona y tarde en el Espirizu Sarto.

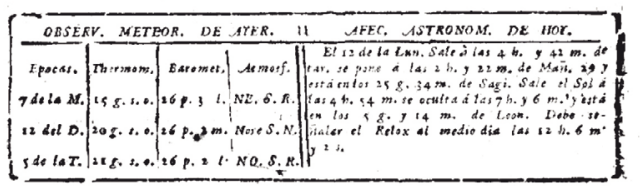

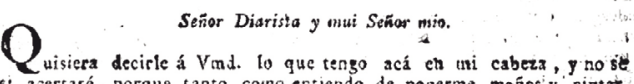
st acertaré, porque tanto cono entiendo de ponerme mañns' y cintaş, etro tanto ignoro de que manera he de explicar mis pensamientos para que ime entiendun las gentes, y no se fastidien de mis palabras. Ab! si lo que escribo me to oyesea de mis labios, bien scgura estoi dé que ao babria un solo humbre, por severo filosofo quic fucse, à quiea no naerectese muchos elogilos: porque ha de saber Vm.t. que is natutal proporcion las harices, la boca; ios ojos, y las cejas; que corr razon se puede decir que me hizo hermosa. Asi lo dicen tudos, asi es:

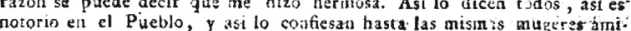
gas anias, quienes no pueden distralay ni su idmiracion, ni sa $\mathrm{tri}$ vidia, quando me miran atentas. Por. io imisno soy mui admitalla de quantus tne tratan, y me han puesto las continuadas lisonjas tn uir esrado, que no puedo sufric que ine contradigan, $n$ i que ho ensalcen hasta los Cielos qualquiera cosa que me pertenezca. belleza que D. Isidro Calle Bcieca puso tu su Diario de Yind. I con especialidad aquelio de to ultimo en qué nos deciza que coto quanto tenemos de hernosas las mugeres se lo debemos a sus mercedes los Seäorss hombres. Yo me creia que inmediatamente hubiesen' llovido los. tas y discursos defendiendo nuestra justicia, y que al St. Trotca to.

(*) Veanse los Diàrios de 30 de Abril, y a de Mayo de este año. 
La carta de la Defensora se iniciaba con una divertida introducción en la que esta se describía a sí misma como una mujer madrileña (la Matallana también lo era) muy hermosa. Su modo de vida aristocrático -confesaba pasarse los días del tocador a la tertulia, y de la tertulia al paseo- y sus costumbres refinadas la calificaban como una perfecta petimetra de la nobleza. El texto continuaba con una dura crítica al sexismo imperante en la sociedad española de la época, donde se acusaba a los hombres de mantener a las mujeres en un estado de total exclusión de la vida pública ${ }^{44}$. Tras una serie de digresiones, el texto se centraba en la crítica al relativismo estético defendido por Ceballos. La Defensora de la belleza insistía en la existencia de una belleza esencial como atributo de la naturaleza, así como en la superioridad estética de las mujeres europeas. Incidía en señalar los defectos de los argumentos relativistas defendidos por Ceballos, con irónicas alusiones ad hominem al interés que el oficial cántabro había mostrado hacia las nativas de pueblos no europeos a lo largo de la expedición científica alrededor del mundo. Tanto en esta como en otras cartas de Malaspina al Diario de Madrid, abundan las alusiones a los contactos de Ceballos con las mujeres nativas durante la expedición:

Acompáñese el Sr. Boceca de las damas que producen las heladas regiones de los polos, recréese con sus bocas rasgadas hasta las orejas ${ }^{45}$, con sus narices aplastadas, con sus ojos pajizos, con sus pellejos de baqueta de Moscovia, y sus colores de cobre y aplomados. ${ }^{46}$

Para la Defensora de la belleza, no cabían dudas sobre la superior belleza de los pueblos europeos y, dirigiéndose siempre a Boceca/Ceballos, le interpelaba con sorna: “¿Cómo quiere Vmd. encontrar el bello esencial si se va a buscarlo entre las naciones salvajes?"47. Por su parte, Malaspina y la Matallana, escondidos tras su disfraz de la Defensora, se mostraban absolutamente convencidos de que "en la Grecia, en la Italia, y en lo meridional de nuestra península (...) los hombres y las mujeres [constituían] las producciones más perfectas de la naturaleza" 48 .
Fig. 2. Mujer de Mulgrave. Reproducido con permiso del $\mathrm{Mu}$ seo Naval de Madrid. En su carta publicada en el Diario de Madrid de 1795, Malaspina y la Matallana se burlaban del gusto de Ceballos por las mujeres no europeas: “Acompáñese el Sr. Boceca de las damas que producen las heladas regiones de los polos, recréese con sus bocas rasgadas hasta las orejas" Diario de Madrid, (30/07/1795).

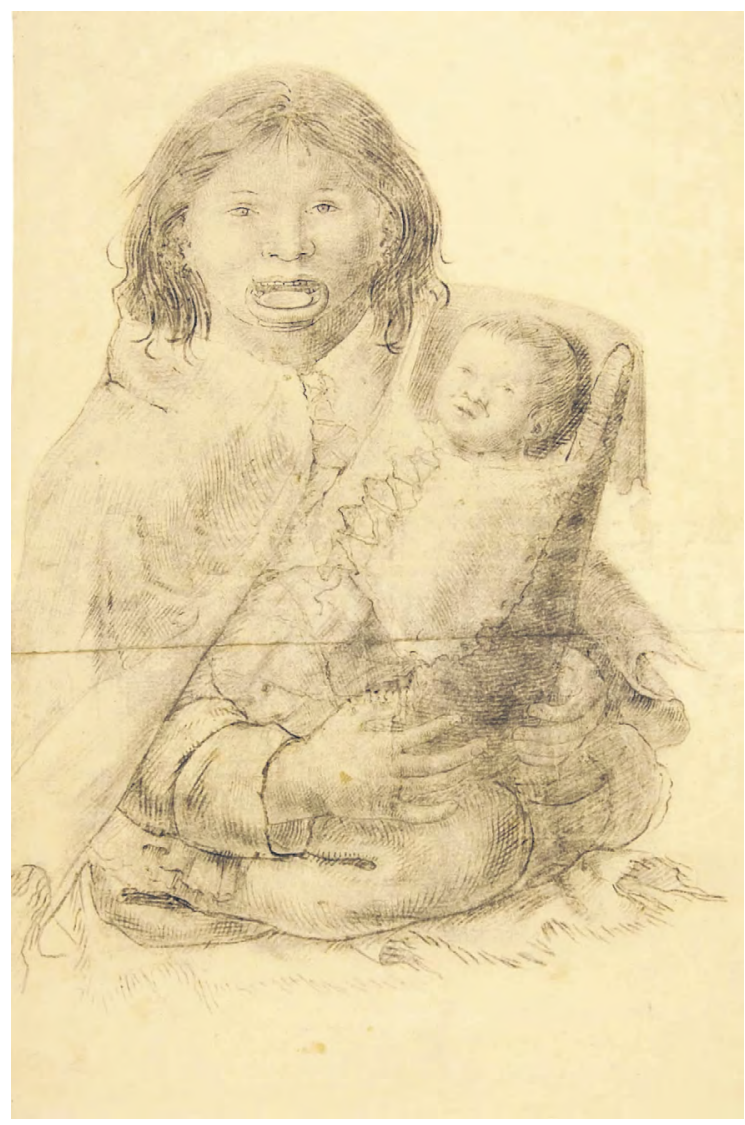

Poco después de coescribir la carta de la Defenso$r a$, la marquesa fue nombrada oficialmente dama de cámara de la Reina María Luísa, lo que facilitó aún más su capacidad de acceso a los monarcas ${ }^{49}$. La Matallana, aficionada, como sabemos, a las maquinaciones palaciegas, no podía dejar pasar esa oportunidad para renovar sus intentos de derrocar a Godoy ejerciendo su influencia sobre la Reina, de quien se encontraba más cerca que nunca. Aquel divertimento filosófico sobre la belleza que el brigadier y la marquesa escribieron a dos manos a fines de Julio de 1795 en el Diario de Madrid pudo perfectamente servir de inspiración para que tan solo unos pocos meses después, Fernanda O'Connock, ya con pleno acceso a la cámara real, volviera a actuar como amanuense del italiano -para no dejar pistas con su letra y con su peculiar sintaxisen el plan para instaurar un nuevo gobierno, con el que Malaspina pretendía encarcelar a Godoy en la Alhambra de Granada y mudar para siempre el destino de 
España y sus colonias. Dados los antecedentes de la Matallana, resulta verosímil pensar que, como en el caso de Picornell, Malaspina se dispuso a actuar creyendo contar con el apoyo, por mediación de su amiga la marquesa, de importantes personajes de la oposición interna.

A la luz de estos hechos, lo más probable es que, aprovechando uno de los frecuentes accesos de celos de la monarca hacia Godoy, la marquesa convenciera a la reina de que su íntimo amigo era el hombre ideal para concebir un plan de gobierno que de una vez por todas librara a España del sultán. La reina, despechada, aceptó la sugestión de su dama de confianza y, bajo el máximo secreto, encargó a Malaspina que redactase su plan $^{50}$. Inicialmente, Malaspina recusó el pedido, a sabiendas de que se jugaba el tipo. Sin embargo, finalmente acabó aceptando el encargo real ${ }^{51}$. Lo pagó caro, con más de seis años de aislamiento en un islote gallego, donde tuvo tiempo suficiente para recordar las tertulias eruditas de la corte, su disputa en el Diario de Madrid y continuar meditando sobre la belleza.

Fig. 3. Advertencia preliminar del manuscrito de la Meditación filosófica de Malaspina, en la que rememora su participación en la Disputa literaria por la Belleza que tuvo lugar en el Diario de Madrid a lo largo de 1795 . En este texto, escrito en prisión tras el fracaso de su intentona de derrocar a Godoy, Malaspina retomó los mismos temas que había planteado meses antes en sus cartas al Diario de Madrid. El Manuscrito original se conserva en el Centro di Studi Malaspiniani de Mulazzo (Italia).

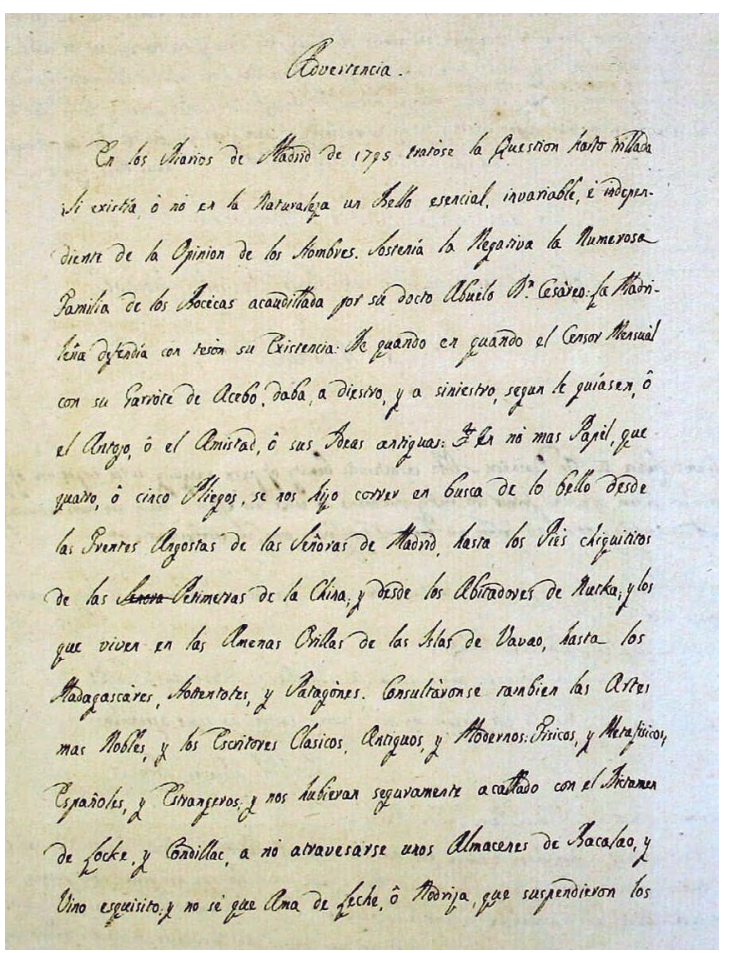

\section{AGRADECIMIENTOS}

Agradezco al Dr. Andrés Galera por su apoyo durante mi estancia en el Centro de Ciencias Humanas y Sociales del CSIC entre feb. de 2019 y enero de 2020. Agradezco a los colegas del Dpto. de Estudios Americanos y del Dpto. de Historia de la Ciencia del CSIC, y en especial a los doctores Juan Pimentel, José Luis Peset y Fermín del Pino por sus comentarios y preguntas en el seno del Seminario de Historia de la Ciencia del CSIC, donde presenté los resultados principales de este trabajo en diciembre de 2019. También agradezco a los revisores anónimos de este trabajo, quienes con sus acertados comentarios y sugerencias ayudaron a mejorar la calidad del artículo. Por último, agradezco a la Coordenação de Aperfeiçoamento de Pessoal de Nível Superior - Brasil (Capes) por su apoyo a este trabajo entre octubre de 2019 y enero de 2020- Código de Financiación 001.

\section{NOTAS}

1 Malaspina afirmaba que, "tal vez no sería aventurado el asegurar que las colecciones formadas en el viaje son las más selectas que existan en el día, por sus rarezas, variedad y número", Novo y Colson (ed.) (1885), p. 46. En el presente trabajo, todas las citas del viaje de Malaspina han sido extraídas de la edición de Novo y Colson (1885). En 1987 apareció una nueva edición del viaje en 9 tomos, Higueras (coord.) (1987-99). En 2001 la Hakluyt Society emprendió la publicación completa del viaje en inglés, y en 2018 lanzó su segunda edición, David et al. (eds). (2018).

2 Galera (1988). Sobre la labor en el viaje de los naturalistas Tadeo Haenke y Luis Nee, Véase Ibáñez (1992); Galera, (ed.) (2016).

3 En este grupo selecto se encontraban Bustamante, Alcalá Galiano, Ciriaco de Ceballos, Felipe Bauzá, Juan Vernacci, Jacobo Murphy, y Fabio Ala Ponzone, así como el naturalista Luis Née y los pintores Ravenet y Bambrila. Véase Manfredi (1999), p. 75, nota 151.

4 Sobre el pensamiento político de Malaspina, véase Pimentel (1998); Lucena y Pimentel (1991).

5 Novo y Colson, (ed.) 1885, p. xxx.

6 Manfredi, (1999), p. 315

7 Manfredi (1994), p. 99

8 Novo y Colson (ed.) (1885), p. 43.

9 «Dos reflexiones de la mayor entidad son las que nos vienen al encuentro (...); la primera, si efectivamente el tratar de la emancipación de las colonias, divididas en los tres grandes trozos o confederaciones que se han indicado, es una proposición en realidad tan odiosa y temible cual lo parece a primera vista; la segunda, si el tratado propuesto puede o debe sin riesgo alguno ser público para toda la Nación y aún para las demás naciones, o émulas o superiores a nosotros en fuerzas». Novo y Colson (ed.) (1885), p. XXXI.

10 Novo y Colson (ed.) (1885), p. 43 
11 Manfredi (1994), p. 325.

12 Esta incontinencia verbal de Malaspina no pasó desapercibida a sus amigos de aquel periodo. A finales de 1.794, Juan Peña y Ruiz, amigo de Alejandro, se hacía eco de su imprudencia al hablar abiertamente sobre los males del estado y las reformas indispensables para sanar esos males. En una carta a su común amigo Greppi, Peña afirmaba que "Malaspina ha viajado por los mares y ha perdido de vista la tierra". Manfredi (1994), p. 103.

13 "Cada día siento más antipatía por el oficio de autor», confesaba Alejandro a Paulo Greppi en una carta escrita en Aranjuez el 26 de mayo de 1795. Cf. Manfredi (1994), p. 345. Unas semanas después escribía a Ramón Ximénez: "Cada día me parece más difícil y pesada la carga de escribir». Véase Manfredi (1999), p. 353.

14 A la humillante designación de Manuel Gil como director y principal redactor de la relación del viaje de Malaspina había que sumar -absorbidas las rentas del estado por la guerra con Francia- el problema de la falta de caudales. Finalmente, los asuntos del erario acabaron siendo determinantes e impidieron publicar el viaje. Cf. Marín-Merás Verdejo (2016), p. 42.

15 Gil describía el comportamiento de aquellos días de Malaspina como el de alguien afectado por una "insanable comezón política que hacía que apenas podía moverse sin referirse al comercio, la industria, a las relaciones con los pueblos de más ramos de la legislación, con la aplicación de todo esto a las Américas". Cf. Manfredi (1994), p. 103.

16 En febrero de 1795 Alejandro dio por primera vez muestras de esa fatídica imprudencia ante Godoy al presentarle -por intermediación del ministro Valdés- un plan personal para firmar la paz con Francia. Esto levantó las sospechas de Godoy, quien tras leer el plan preguntó a Valdés si su protegido había perdido la cabeza. Cf. Giura Longo y Rossi (eds.)(1999), p. 221.

17 Jiménez de la Espada (1881), p. 402.

18 Ibidem.

19 Valdés llegó a afirmar que «por sus conocimientos, cuna, nobleza y elegancia de la persona y maneras, arrogante presencia, afabilidad, firmeza de carácter y talento de sociedad, era Malaspina el primero de nuestra Armada». Fernández Duro (1902), p. 53-54.

20 Sobre el clérigo sevillano Manuel Gil, Cf. Soler Pascual (1993), pp. 261-287.

21 Véase Sotos Serrano (1982).

22 Sobre Fabio Ala Ponzone, Véase Manfredi, (ed.) (1999).

23 Véase Gutiérrez de la Concha (1791), p. 166.

24 Véase Viana (1993), p. 226.

25 Véase Ceballos (1993); Bustamante (1989).

26 Véase un sumario de todas las cartas de la Disputa sobre la belleza en la TABLA 1.

27 Fernández y Manfredi (1998). Si mi análisis es correcto, el texto atribuido a Malaspina por Fernández y Manfredi fue redactado por Ciriaco de Cebayos. Véase TABLA 1, CARTA №23(10-11/09/1795), nota 3.

28 Pero, como en el caso de Malaspina, la Disputa por la Belleza contiene otras cartas de Gil. Véase la TABLA 1.

29 Black y Clemotte-Silvero (2007).
30 Sobre la "conspiración de Malaspina", Véase Manfredi (1987); Soler Pascual (1990); Beerman (1992).

31 Véase Jiménez de la Espada (1881), 402.

32 Llamada así para distinguirla de la "oposición externa", o republicana, que contaba con el apoyo de Francia, y que actuaba difundiendo clandestinamente en el país propaganda pro-revolucionaria. Véase Aguirrezábal y Comellas (1982). Sobre los movimientos sediciosos de oposición al régimen en tiempos de Godoy, véase Elorza (ed.) (1971).

33 Existen indicios de que también Malaspina perteneció a la masonería. Poco tiempo antes de ser detenido, en septiembre de 1795, Malaspina escribía al masón Ramón Ximénez de Cenarbe, preceptor de su discípulo Fabio Ala Ponzone, confiándole que, cuando Fabio retornara a Italia esperaba que Ximénez pudiera "lavar en las aguas del Jordán a Fabio", lo que entiendo como una referencia a su iniciación ritual en alguna logia. Véase Manfredi (ed.) (1999), p. 362. Sobre Ramón Ximénez de Cenarbe (1743-1831), Véase Rangognini (2002).

34 Véase Aguirrezábal y Comellas (1982), p.13.

35 La Marquesa vivía en una casa contigua a la residencia de Malaspina, que se hospedaba en la casa del príncipe de Monforte, en los aledaños del palacio de los duques de Alba, hoy cuartel general de ejército. Véase Jiménez de la Espada (1881), p. 411.

36 El propio Conde de Aranda "participaba activamente en tertulias ideológico políticas presididas simbólicamente por damas: La Condesa de Montijo, la Marquesa de Matallana, la Condesa Duquesa de Benavente, etc., en las que el prócer aragonés era el alma de la reunión". Aguirrezábal y Comellas (1882), pp. 32-33.

37 Beerman (1992), p. 102.

38 Greppi, (1883), p. 46.

39 Aguirrezábal y Comellas (1982), p. 32.

40 En el contexto de la Disputa sobre la belleza, las alusiones al cortejo de Malaspina a la Matallana son especialmente claras en las cartas de Manuel Gil. Véase la TABLA 1.

41 Aun así, considero probable que el cortejo de Malaspina no tuviera un carácter sexual. Existen claros indicios de que el italiano era homosexual. En su Meditación filosófica, Malaspina no sólo hizo una decidida defensa de la homosexualidad en el ejército espartano (Meditación filosófica, hoja no 41), sino que reconoció abiertamente que, a su juicio, el ser más hermoso de la tierra era el famoso torero español Pedro Romero. Meditación filosófica, Nota Y, hojas no 126-127. La presunta homosexualidad de Malaspina ya había sido el objeto de algunos chascarrillos entre los especialistas. Manfredi y Sáiz (1995), reconocían haber escuchado comentarios entre malaspinistas, aludiendo a "una cierta indiferencia (por no decir otra cosa) en relación al "bel sesso" " Manfredi y Blanca Sáiz (1995: 298). Sin embargo, estos autores restaban valor a esos rumores, dando por hecho que la relación amistosa entre Barbara Litta y el italiano tuvo un cariz erótico, algo que, a la luz de la Meditación filosófica, me permito poner en duda. De igual forma, considero inverosímiles las alusiones al supuesto "romance» entre Alejandro y la marquesa de Matallana, que Soler (1990) aceptaba «sin ninguna duda», y que Manfredi consideraba probable. A mi juicio -e independientemente de los sentimientos que Barbara Litta o la marquesa de Matallana (ambas, mujeres casadas) pu- 
dieran haber albergado hacia el puritano Alejandro-, tales relaciones consistieron apenas en "cortejos» de carácter amistoso -una costumbre plenamente aceptada entre la aristocracia madrileña de fin de siglo, $c f$. Towsend (2004) pp. 343-346. En este sentido, considero que la interpretación más verosímil de la relación entre Malaspina y sus amigas casadas la aporta Greppi, quien aseguraba que la relación entre Alejandro y la Matallana fue siempre, y a pesar de los rumores, «inocentissima» cf. Greppi (1883: 33-57).

42 Cf. Towsend (2004) pp. 343-346.

43 Véase el Diario de Madrid de los días 28, 29 y 30 de julio de 1795.

$44 \mathrm{El}$ alegato feminista del texto de la Defensora es mucho más radical que el de la Meditación filosófica, por lo que resulta verosímil suponer que en esta parte la marquesa llevó "la voz cantante" en la redacción.

45 Las mujeres de Mulgrave, uno de los puntos más cercanos al polo donde recaló la Expedición Malaspina, tenían por costumbre horadar sus labios. Véase la fig.2.

46 Diario de Madrid (30/07/1795).

47 Diario de Madrid (29/07/1795).

48 Diario de Madrid (30/07/1795). Esta idea se corresponde perfectamente con lo expresado por Malaspina en su Meditación filosófica.

49 Fue nombrada Dama de Corte el 4 de septiembre de 1795. Véase Jiménez de la Espada (1881), p. 411, nota 1

50 Beerman (1992) suponía que Malaspina había dictado sus planes de reforma de gobierno al padre Gil, pero es un error que puede descartarse. En una carta del 24 de diciembre de

\section{BIBLIOGRAFÍA}

Aguirrezábal, María Jesús; Comellas, José Luis (1982), “La conspiración de Picornell (1795) en el contexto de la prerrevolución liberal española", Revista de historia contemporánea, 1, pp. 7-38.

Beerman, Eric (1992), El diario del proceso y encarcelamiento de Alejandro Malaspina, 1794-1803, Madrid, Editorial Naval.

Black, John; Clemotte-Silvero, Oscar (2007), A translation of Alexandro Malaspina's Meditación sobre lo Bello en la Naturaleza, Queenston, The Edwin Mellen Press.

Bustamante, Jesús (1989), "Los vocabularios mexicanos, Malaspina, y la Costa Noroeste." En: Peset, José Luis, (ed.) (1989) Culturas de la Costa Noroeste de América, Madrid, Turner, pp. 81-92.

Ceballos, Ciriaco de (1993), "Vocabulario de Vavao, por el Teniente de navío de la Real Armada D. Ciriaco Cevallos", 1793. En: Higueras Rodríguez, Ma Dolores; Pimentel Igea, Juan (eds.), (1993) La expedición Malaspina, 1789-1794, Vol. V, Atropología y Notícias Etnográficas, Madrid, Ministerio de Defensa/Museo Naval/Lunwerg, pp. 227-41.

David, Andrew; Fernández-Armesto, Felipe; Williams, Glyndwr, (eds.) (2018), The Malaspina Expedition 1789-1794: Journal of the Voyage by Alejandro Malaspina, 3 vols, London, Routledge/Hakluyt Society.
1.810, el amigo de Malaspina Lazzaro Brunetti afirma que Alejandro le confesó personalmente antes de morir que la propia Reina le pidió que redactase un programa de renovación radical. Véase Manfredi (1986), p. 27. La tesis de que la Reina pidió a Malaspina que le entregase un plan de reforma gubernamental por escrito, también fue corroborada por Emmanuele Greppi -nieto del íntimo amigo de Alejandro, Paolo Greppi- y por el historiador, masón, y diputado liberal Lorenzo Villanueva, testigo de aquella época quien resumió así lo acontecido: "Lo que parece más verosímil, o, por mejor decir, se tiene por cierto, es que aquel célebre marino fue víctima de un enredo entre la Reina y dos damas suyas, la Matallana y la Pizarro, y el Príncipe de la Paz. En un intervalo de desafecto y resentimiento, en cuyo tiempo andaba la Reina a caza de medios para cortar la privanza del valido, fue buscado Malaspina por estas damas para que (..) trajera realizado el plan". Villanueva (1825), p. 55. María Pizarro fue otra de las Damas de la Reina que actuaba como espía de Godoy y acabó dando al traste con los planes de Malaspina y la Matallana. Malaspina confió en ella ignorando que la Pizarro era espía de Godoy. Esta, apenas tuvo en su poder los documentos que le confió Malaspina para hacérselos llegar al confesor del rey, se los entregó al valido, precipitando la detención del italiano y de la Matallana.

51 "La Reina pidió entonces que Malaspina extendiese un nuevo plan de gobierno al Ministerio. Él se negaba, pero finalmente condescendió, afirmando sin embargo que sería sacrificado por ello, pues la reina no tenía suficiente firmeza". Véase Greppi, (1883), p. 46.

Elorza, Antonio (ed.) (1971), Pan y toros y otros papeles sediciosos de fines del siglo XVIII, Madrid, Ayuso.

Fernández, Belén; Manfredi, Dario (1998), “Un ignorato scritto estético di Alessandro Malaspina in una disputa sopra la Belezza (1795)", Archivio Storico per le provincie parmensi, Quarta serie, vol. XLIX, 75-103.

Fernández Duro, Cesáreo (1902), Armada Española desde la unión de los reinos de Castilla y Aragón, Vol 8, Madrid, Sucesores de Ribadeneyda,

Galera, Andrés, (ed.) (2016), El Arca de Neé: plantas recolectadas por el botánico Luis Neé durante la Expedición Malaspina, Madrid, Consejo Superior de Investigaciones científicas.

Galera Gómez, Andrés (1988), “Las ciencias naturales en la expedición Malaspina (1789-1794): la labor científica de Antonio Pineda y Ramírez", Tesis doctoral, Madrid, Universidad Complutense de Madrid.

Galera Gómez, Andrés; Peralta, Víctor, (eds.) (2016), Historias Malaspinianas, Madrid, CSIC.

Giura Longo, Raffael; Rossi, Pasquale (eds.) (1999), Con Malaspina nei Mari del Sud. Bari, Ed. B.A. Graphis.

Greppi, Emanuele (1883), "Un italiano alla corte di Spagna nel secolo XVIII. Alessandro Malaspina", Nuova Antologia, II (XXXVIII), pp. 33-57. 
Gutiérrez de la Concha, Juan (1993 [1791]), Extracto de los sucesos acaecidos en el reconocimiento de la costa NO de América en 1791 por Juan Gutiérrez de la Concha, oficial de la corbeta Atrevida. En: Higueras y Pimentel (eds.) (1993), La expedición Malaspina, 1789-1794, Vol. V, Atropología y Notícias Etnográficas, Madrid, Ministerio de Defensa/Museo Naval/Lunwerg, pp. 153-168.

Higueras Rodríguez, Ma Dolores, (coord.) (1987-99), La Expedición Malaspina, 1789-1794, 9 vols, Madrid, Museo Naval-Ministerio de Defensa/Lunwerg,

Ibáñez, Victoria (1992), Trabajos científicos y correspondencia de Tadeo Haenke. Madrid, Museo Naval/Lunwerg.

Jiménez de la Espada, M. (1881), "Una causa de estado”, Revista Contemporánea, 4, (31), pp. 401-404; (32), pp. 279-305; (33), pp. 401-34

Lucena Guiraldo, Manuel; Pimentel, Juan (1991), Los "Axiomas políticos sobre la América" de Alejandro Malaspina, Madrid, Doce Calles.

Manfredi, Dario, (ed.) (1999), Alessandro Malaspina e Fabio Ala Ponzone. Lettere dal Vecchio e Nuovo Mondo (1788-1803), Bologna, II Mulino.

Manfredi, Dario (1994), "Alejandro Malaspina. Una biografía”. En: Saiz, Blanca (ed.), Alejandro Malaspina. La América imposible, Madrid, Compañía literaria, pp. 19-135.

Manfredi, Dario (1987), “Il ritorno a Espagna. L'arresto, la prigionia". In Alessandro Malaspina nella geografía del suo tempo, Genova, Civico Istituto Colombiano, 161-185.

Manfredi, Dario (1986), Sugli anni "pontremolesi" di Alessandro Malaspina, 1803-1810, La Spezia, Centro “Alessandro Malaspina" per la Storia e le Tradizioni marinare.

Manfredi, Dario; Sáiz, Blanca (1995), “"Callejones sin salida...” Tracce di una relazione sentimentale di Alessandro Malaspina». En: Blanca Sáiz, (ed.), Malaspina 93. Alessandro Malaspina e La sua spedicizione scientifica 1789-1794. Atti del Congesso Internazionale nel bicentenário della massima impresa di Alessandro Malaspina. Mulazo-Castiglione Del Terziere-Lerici
24-26 settembre 1993, Mulazzo, Centro Alessandro Malaspina per La storia e le tradizione marinare, pp. 297-315

Marín-Merás Verdejo, Luisa (2016), “La expedición Malaspina: un epílogo". En: Galera, Andrés; Peralta, Víctor (eds.) Historias Malaspinianas, Madrid, CSIC, pp. 23-47.

Novo y Colson, Pedro (1885), Viaje político-científico alrededor del mundo por las corbetas Descubierta y Atrevida, Madrid, Viuda e hijos de Abienzo.

Pimentel, Juan (1998), La física de la monarquía: ciencia y política en el pensamiento colonial de Alejandro Malaspina (17541810), Madrid, Doce calles.

Rangognini, Enzo (2002), L'istitutore aragonese: lettere di Ramón Ximénez de Cenarbe a Fabio Ala (1787-1815), Cremona, Linograf.

Soler Pascual, Emilio (1993), "Noticia del Padre Manuel Gil, de los clérigos menores de Sevilla". En Malaspina 93. Alessandro Malaspina e la sua spedicizione scientifica 1789-1794. Atti del Congesso Internazionale nel bicentenário della massima impresa di Alessandro Malaspina, Mulazo-Castiglione de Terziere-Lerici, Centro Alessandro Malaspina per la storia e le tradizione marinare, pp. 261-287.

Soler Pascual, Emilio (1990), La conspiración Malaspina (17951796), Alicante, Instituto de cultura "Juan Gil-Albert".

Sotos Serrano, Carmen (1982), Los pintores de la expedición Malaspina, Madrid, Real Academia de la Historia.

Towsend, Joseph (2004 [1791]), A Journey Through Spain in the Years 1786 and 1787, 3 vols., Londres, Dilly. En: Thomas, Hugh (2004) Antología de Madrid, 343-46, Madrid, Gadir.

Viana, Francisco Javier (1993), "Estada en el Archipiélago de Mayorga o islas de Vavao" En Higueras Rodríguez, Ma Dolores, y Pimentel Igea, Juan (eds.) (1993), La expedición Malaspina 1789-1794, Madrid, Ministerio de Defensa/Museo Naval/ Lunwerg, pp. 211-26

Villanueva, Lorenzo (1825), Vida literaria de don Joaquín Lorenzo Villanueva, Londres, Dulau \& Co. 